\title{
Complicaciones neurológicas en pacientes adultos sometidos a trasplante hepático ortotópico. Experiencia de un centro universitario
}

\author{
Patricio Mellado ${ }^{1}$, Pilar Peredo ${ }^{1}$, Raúl Valenzuela ${ }^{1}$, \\ Marco Arrese ${ }^{2}$, Rosa María Pérez ${ }^{2}$, Pilar Domíngueza \\ Juan Francisco Guerra ${ }^{3}$, Nicolás Jarufe ${ }^{3}$, Jorge Martínez ${ }^{3}$. \\ Neurological complications \\ following a liver transplantation. \\ Experience from a Chilean center
}

Background: Orthotopic liver transplantation (OLT) is the treatment of choice for multiple acute and chronic end-stage liver diseases as well as for selected cases of liver malignancy and liver-site metabolic disorders. Neurological impairment is a major source of morbidity and mortality following OLT. Aim: To describe the incidence and the type of neurological complications occurring in the post-operative period of OLT in patients transplanted in our hospital. Material and methods: Between March 1994 and August 2007, 76 adult patients underwent OLT. Data on incidence, time of onset, and outcome of central nervous system (CNS) complications have been obtained from our program data base and patient charts. Results: Twenty three patients (30.3\%) had CNS complications following OLT. The leading complications were immunosuppressive drug-related neurological impairment in nine patients (39.1\%), peripheral nerve damage in five patients $(21.7 \%)$, central pontine myelinolysis in four patients (17.4\%), cerebrovascular disease in three (13\%) and CNS infection in three (13\%). Most CNS events (90\%) occurred in the first 2 weeks after OLT. Five patients with neurological complications died (22\%). Conclusions: CNS complications occurred in almost one fifth of the population studied, and they had a poor outcome, as previously reported (Rev Méd Chile 2008; 136: 1255-63).

(Key w ords: Cerebrovascular disease; Liver transplantation; Myelinolysis, central pontine)

Recibido el 24 de marzo, 2008. Aceptado el 7 de julio, 2008.

${ }^{1}$ Departamentos de Neurología, ${ }^{2}$ Gastroenterología y ${ }^{3}$ Cirugía Digestiva, Hospital Clínico, Facultad de Medicina, Pontificia Universidad Católica de Chile. Santiago de Chile aEnfermera Universitaria, Coordinadora del Programa de Trasplante Hepático.

Correspondencia a: Dr. Patricio Mellado. Marcoleta 352 Santiago. Santiago. Chile. Fax: 56-2-632-6221. E mail: pmellado@med.puc.cl 
$\mathrm{E}^{1}$ trasplante hepático ortotópico (THO) constituye el tratamiento definitivo de enfermedades hepáticas crónicas terminales, de las formas graves de insuficiencia hepática aguda y de algunas enfermedades metabólicas y neoplásicas. Sus inicios se remontan a 1963 con Starzl ${ }^{1,2}$. En 1983 se aceptó en Estados Unidos de Norteamérica como una opción terapéutica en el tratamiento de enfermedades hepáticas terminales ${ }^{3}$.

El primer intento de THO en Chile fue realizado en 1969 por Bitrán, con muerte inmediata del paciente $^{4}$. En 1985, Hepp realizó el primer THO con sobrevida del paciente ${ }^{5}$. En marzo de 1994 se realizó el primer THO de nuestro programa ${ }^{6}$.

Existen complicaciones que pueden ocurrir después de un THO. Las neurológicas destacan por su cuadro clínico alarmante y su impacto en la sobrevida, recuperación y calidad de vida del paciente ${ }^{7}$.

El objetivo de este trabajo es describir la incidencia y tipo de complicaciones neurológicas (CN) posteriores a los THO realizados en el Hospital Clínico de la Pontificia Universidad Católica de Chile (PUC).

\section{MATERIAL Y MÉTODO}

Estudio descriptivo y retrospectivo a partir de la base de datos prospectiva del programa de THO y de los registros clínicos de los pacientes sometidos a THO en el periodo entre marzo de 1994 y agosto de 2007. Se incluyó sólo a los pacientes que fueron evaluados por el equipo de neurología de adultos, excluyéndose a los menores de 15 años.

La base de datos fue creada desde la cuarta intervención en forma prospectiva, se consignó: la demografía, datos clínicos y de laboratorio de donantes y receptores, tipos de procura realizadas, tiempos de isquemias, transfusiones, detalles técnicos de la intervención, tipos de profilaxis antiinfecciosa, esquemas de inmunosupresión, reintervenciones, días de permanencia en la unidad de cuidados intensivos, mortalidad y causa, anatomía patológica, seguimiento y complicaciones médicas y quirúrgicas ocurridas.

En todos los pacientes se utilizó un esquema inmunosupresor bi o triasociado sobre la base de un anticalcineurínico (ciclosporina o tacrolimus) y esteroides, y en los esquemas triasociados un antiproliferativo (azatioprina o micofenolato mofetil). Inicialmente se utilizó medición de niveles plasmáticos valle (predosis matinal) de ciclosporina $\left(\mathrm{C}_{0}\right)$ y desde el año 2002 se incorporó la medición de niveles plasmáticos $2 \mathrm{~h}$ posteriores a la administración de la dosis matinal $\left(\mathrm{C}_{2}\right)$.

En este estudio se analizó específicamente los siguientes datos: sexo, edad al momento del trasplante, indicación de trasplante, ocurrencia y naturaleza de complicaciones neurológicas, estadía hospitalaria, mortalidad específica, esquema inmunosupresor y el tipo de medición de niveles de inhibidores de calcineurina. Por el tamaño de la muestra los datos numéricos se expresaron en medianas, con su respectivo valor mínimo y máximo. Se usó la prueba de Wilcoxon como indicador estadístico de significación.

Se identificaron todos los pacientes que presentaron CN en su periodo post operatorio y hasta el alta hospitalaria. Todos fueron evaluados por un neurólogo de la PUC y estudiados con imágenes del sistema nervioso central (SNC) y exámenes electrofisiológicos según necesidad.

Las CN se clasificaron en: 1) Efectos secundarios a drogas inmunosupresoras. 2) Enfermedad cerebrovascular. 3) Infecciones del SNC. 4) Mielinolisis pontina (MP). 5) Lesión de sistema nervioso periférico (SNP).

Detalles de la técnica quirúrgica del donante y del trasplante han sido descritos precedentemente ${ }^{6,8}$.

\section{Resultados}

En el período estudiado, 76 pacientes adultos fueron sometidos a un THO, dos pacientes fueron retrasplantados. La indicación de trasplante se resume en la Tabla 1. La mediana de la edad fue 51 años, con una mínima de 15 y máxima de 71 años. En 77,6\%, la indicación fue electiva y en $22,4 \%$, urgente. En los pacientes con daño hepático crónico (DHCr) la función hepática fue evaluada según la clasificación de Child-Turcotte-Pugh ${ }^{9}$.

Las CN se presentaron en 23 pacientes (30,3\%). Uno de ellos presentó dos CN. Ninguno de los pacientes retrasplantados desarrolló una CN. Las características generales de este grupo se detallan en la Tabla 2. De estos pacientes, 10 eran mujeres y 13 hombres. La edad de los pacientes no fue un 
Tabla 1. Etiología de la indicación del T H 0 en pacientes adultos

\begin{tabular}{|lcc|}
\hline Diagnóstico & número de pacientes & $\begin{array}{c}\text { número de pacientes } \\
\text { CN v/s SCN }\end{array}$ \\
\hline Daño hepático crónico & 64 & \\
- Hepatitis autoinmune & 10 & $3 / 7$ \\
- Hepatitis C & $13(2)^{*}$ & $2 / 11$ \\
- Daño hepático crónico por alcohol & $11(2)^{*}$ & $2 / 9$ \\
- Enfermedades por depósito & 5 & $3 / 2$ \\
- Cirrosis biliar primaria & 8 & $2 / 6$ \\
- Neoplasias & $3(2)^{*}$ & $1 / 2$ \\
- Criptogénico & 5 & $3 / 2$ \\
- Esteatohepatitis no alcohólica & $7(1)^{*}$ & $5 / 2$ \\
- Enfermedad de Niemann-Pick & 1 & $1 / 0$ \\
- Vasculitis & 1 & $0 / 1$ \\
Falla hepática aguda fulminante & 12 & \\
- Hepatitis por virus A & 2 & $0 / 2$ \\
- Hepatitis por parvovirus & 1 & $0 / 1$ \\
- Hepatitis secundaria a drogas & 1 & $0 / 1$ \\
- Hepatitis idiopática & 6 & $1 / 5$ \\
- Hepatitis autoinmune & 2 & $0 / 2$ \\
\hline
\end{tabular}

THO: Trasplante hepático ortotópico

*Pacientes con carcinoma hepatocelular concomitante

CN: Complicación neurológica

SCN: Sin complicación neurológica

factor independiente de riesgo para presentar una $\mathrm{CN}$, los que presentaron una $\mathrm{CN}$ tenían una mediana de 53 años [16-65], y los que no la presentaron, una mediana de 51 años [15-71].

Los diagnósticos por los cuales se indicó el THO fueron similares entre el grupo de pacientes que presentó $\mathrm{CN}$ y en los que no las presentaron. Destaca que sólo uno de los pacientes con $\mathrm{CN}$ tenía Child A al momento de THO, 12 Child B y 8 Child C.

La distribución de las $\mathrm{CN}$ se muestra en la Tabla 3. La CN más frecuente fue la toxicidad por drogas inmunosupresoras, que ocurrió en 9 casos (39,1\%). De éstos, cuatro pacientes presentaron un síndrome confusional secundario a ciclosporina, uno sopor y cuatro convulsiones, dos secundarios a ciclosporina y dos a tacrolimus. En promedio, el compromiso de conciencia en estos pacientes se presentó al decimosegundo día postoperatorio (Tabla 4). En todos se realizó una neuroimagen de encéfalo; en 2 de los pacientes que presentaron convulsiones, la resonancia magnética $(\mathrm{RM})$ mostró lesiones hiperintensas córticosubcorticales bilaterales posteriores, compatibles con un síndrome de hiperperfusión cerebral. Los pacientes evolucionaron favorablemente con la suspensión del inmunosupresor y su reemplazo por otro.

De los 7 pacientes que utilizaron ciclosporina y desarrollaron una CN, 6 fueron evaluados con $\mathrm{C}_{0}$ y 1 con $\mathrm{C}_{2}$. El promedio de $\mathrm{C}_{0}$ al momento que los pacientes presentaron toxicidad por ciclosporina fue $363 \mathrm{ng} / \mathrm{ml}$ [220-630]. En cambio, los niveles de quienes no las desarrollaron fue $250 \mathrm{ng} / \mathrm{ml}$ [70350], este último valor fue determinado en base al día promedio en que el primer grupo presentó complicaciones. Esta diferencia fue estadísticamente significativa $(p=0,02)$. Posterior a la incorporación de la medición de niveles $\mathrm{C}_{2}$, sólo un paciente presentó un síndrome confusional agudo, con niveles $\mathrm{C}_{2}$ de $915 \mathrm{ng} / \mathrm{ml}$. 
Tabla 2. Pacientes sometidos a un TH 0 que presentaron complicación neurológica

\begin{tabular}{|c|c|c|c|c|c|c|c|c|c|}
\hline $\mathrm{N}^{\circ}$ & Sexo & Edad & Indicación & Diagnóstico & Complicación neurológica & Pje. & СТР & Día CN & Alta \\
\hline 1 & $\mathrm{~F}$ & 51 & Electiva & Cirrosis biliar primaria & Sopor por ciclosporina & 10 & $\mathrm{C}$ & 4 & V \\
\hline 2 & M & 27 & Electiva & Hepatitis autoinmune & Aspergilosis encefálica & 13 & $\mathrm{C}$ & 30 & $\mathrm{~F}$ \\
\hline 3 & M & 49 & Electiva & Hemocromatosis & SC por ciclosporina & 11 & $\mathrm{C}$ & 17 & V \\
\hline 4 & $\mathrm{~F}$ & 28 & Electiva & Hepatitis autoinmune & Convulsiones por ciclosporina & 8 & B & 7 & V \\
\hline 5 & M & 38 & Electiva & Hepatitis por virus $C$ & Convulsiones por ciclosporina & 9 & B & 17 & V \\
\hline 6 & M & 53 & Electiva & DHCr por alcohol & Hemorragia encefálica masiva & 9 & B & 8 & $\mathrm{~F}$ \\
\hline 7 & M & 51 & Electiva & DHCr criptogénico & Mielinolisis pontina & 9 & B & 4 & V \\
\hline 8 & M & 61 & Electiva & Hemocromatosis & SC por ciclosporina & 9 & B & 15 & V \\
\hline 9 & $\mathrm{~F}$ & 23 & Urgencia & Hepatitis idiopática & SC, aspergilosis encefálica & - & - & 5,21 & $\mathrm{~F}$ \\
\hline 10 & $\mathrm{~F}$ & 51 & Urgencia & DHCr por alcohol & Hemorragia encefálica, convulsión & 12 & $\mathrm{C}$ & 5 & $\mathrm{~V}$ \\
\hline 11 & M & 58 & Electiva & Hemocromatosis & Hemorragia encefálica masiva & 9 & B & 18 & F \\
\hline 12 & M & 16 & Electiva & Niemann Pick tipo B & Síndrome de hiperperfusión cerebral & 8 & $\mathrm{C}$ & 4 & V \\
\hline 13 & M & 55 & Electivo & NASH & Neuropatía cubital compresiva & 8 & $\mathrm{C}$ & 15 & V \\
\hline 14 & F & 59 & Electivo & NASH & Neuropatía peronea compresiva & 9 & C & 17 & V \\
\hline 15 & M & 59 & Electivo & DHCr criptogénico & Mielinolisis pontina & 11 & $\mathrm{C}$ & 12 & $\mathrm{~V}$ \\
\hline 16 & F & 66 & Electivo & Cirmosis biliar primaria & Neuralgia post-herpética & 9 & B & 90 & V \\
\hline 17 & M & 62 & Electivo & NASH & SC por ciclosporina & 11 & C & 6 & V \\
\hline 18 & F & 59 & Electivo & DHCr criptogénico & Mucormicosis encefálica & 6 & A & 60 & V \\
\hline 19 & M & 62 & Electivo & Hepatitis por virus C & Mielinolisis pontina & 11 & $\mathrm{C}$ & 13 & $\mathrm{~V}$ \\
\hline 20 & $\mathrm{~F}$ & 49 & Urgencia & DHCr criptogénico & Polineuropatía del paciente crítico & & - & 10 & $\mathrm{~F}$ \\
\hline 21 & $\mathrm{~F}$ & 64 & Electivo & Hepatocarcinoma & Polineuropatía de fibra fina & 7 & B & 30 & V \\
\hline 22 & F & 28 & Electivo & Hepatitis autoinmune & Síndrome de hiperperfusión cerebral & 9 & B & 20 & V \\
\hline 23 & M & 60 & Electivo & NASH & Mielinolisis pontina & 12 & C & 35 & V \\
\hline
\end{tabular}

THO: Trasplante hepático ortotópico

DHCr: Daño hepático crónico

NASH: Esteatohepatitis no alcohólica

Pje: Puntaje de Child-Turcotte-Pugh al momento del trasplante hepático ortotópico

CTP: Categoría según puntaje de Child-Turcotte-Pugh

CN: Complicación neurológica

SC: Síndrome confusional

V: Vivo

F: Fallecido

Complicaciones cerebrovasculares se presentaron en 3 casos (13\%): dos pacientes con una hemorragia encefálica masiva e hipertensión intracraneana secundaria y una paciente con una hemorragia encefálica de $6 \mathrm{ml}$. Los dos pacientes con hemorragia masiva fallecieron. El primero presentó la hemorragia al octavo día del trasplante, se encontraba normotenso, el recuento plaquetario era $35.000 / \mathrm{mm}^{3}$, el INR 1,8 y el tiempo de tromboplastina parcial activado (TTPA) $28 \mathrm{~s}$. El paciente recibía aspirina (100 mg/día) debido a una trombosis portal pretrasplante.
El segundo paciente presentó coma de inicio súbito al decimoctavo día postoperatorio (Figura 1). El paciente se encontraba normotenso, el recuento plaquetario era $40.000 / \mathrm{mm}^{3}$, el INR 1,3 y el TTPA 23,9 s. El paciente recibía aspirina (100 mg/día) debido a una anastomosis arterial compleja.

La tercera paciente presentó una hemiparesia e hipoestesia izquierda asociada a crisis convulsiva parcial simple al quinto día del trasplante, también se encontraba normotensa, tenía un recuento plaquetario de $35.000 / \mathrm{mm}^{3}$, INR 1,1 y TTPA $25,1 \mathrm{~s}$. Se recuperó sin secuelas. 
Tabla 3. Clasificación de las complicaciones neurológicas

\begin{tabular}{|lc|}
\hline Complicación neurológica & Pacientes (n) \\
\hline Efecto secundario a drogas & 9 \\
- Delirium & 4 \\
- Convulsiones & 4 \\
- Sopor & 1 \\
- Hemfermedad cerebrovascular & 3 \\
- Hemorragia intracerebral masiva & $2^{\dagger}$ \\
Infecciones SNC & $1^{*}$ \\
- aspergilosis encefálica & 3 \\
- mucormicosis & $2^{\dagger}$ \\
Mielinolisis pontica & 1 \\
Lesión de SNP & 4 \\
- Neuralgia post herpética & 5 \\
- Neuropatía compresiva & 1 \\
- Polineuropatía de fibra fina & 2 \\
- Polineuropatía paciente crítico & 1 \\
\hline
\end{tabular}

†Fallecidos

*Paciente con convulsiones concomitantes

SNC: Sistema nervioso central

SNP: Sistema nervioso periférico

Tabla 4. Promedio de días en que se presentaron las complicaciones neurológicas

\begin{tabular}{|lc|}
\hline Tipo complicación & Día postoperatorio \\
\hline Secundario a drogas & 12 \\
- Delirium & 10 \\
- Convulsiones & 12 \\
Enfermedad cerebrovascular & 10 \\
Infección SNC & 37 \\
Mielinolisis pontina & 16 \\
\hline
\end{tabular}

SNC: Sistema nervioso central

Los 3 pacientes que presentaron una hemorragia encefálica se encontraban en tratamiento inmunosupresor con micofenolato en dosis alta para retardar la introducción de ciclosporina, pues tenían factores de riesgo para desarrollar insuficiencia renal. El resto de los pacientes que recibieron micofenolato y que no tuvieron hemorragia encefálica tenía recuento plaquetario mayor de $60.000 / \mathrm{mm}^{3}$.
Infecciones del SNC se presentaron en 3 pacientes (13\%), 2 aspergilosis encefálica y 1 mucormicosis encefálica. Las primeras se diagnosticaron a los $30 \mathrm{y}$ 21 días postrasplante. Uno de estos pacientes presentó además, al quinto día, un síndrome confusional transitorio por ciclosporina. Ambos fallecieron debido a la aspergilosis (Figura 2). El tercer paciente desarrolló una mucormicosis encefálica de origen rinosinusal, las manifestaciones clínicas se iniciaron 


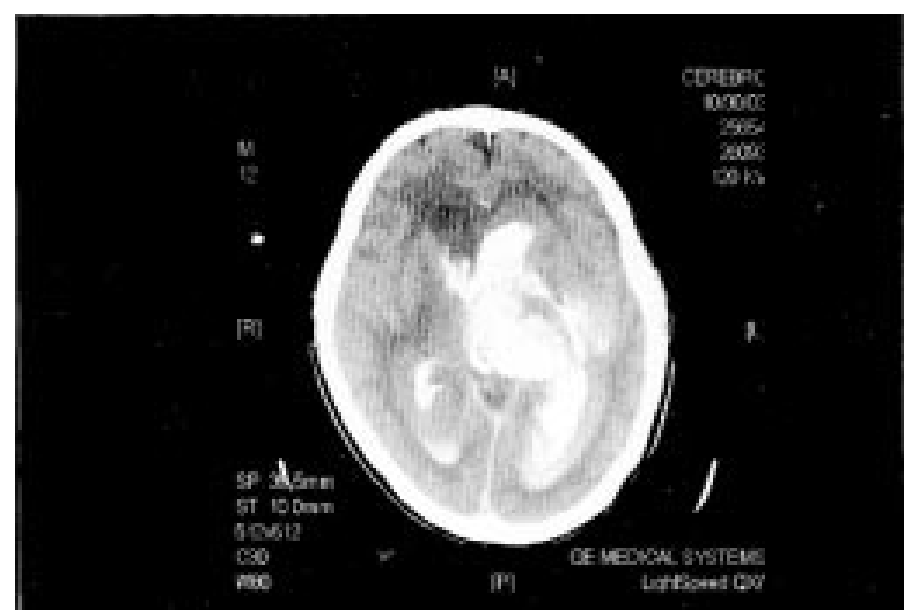

Figura 1. Tomografía computarizada de encéfalo. Hemorragia talámica izquierda masiva con invasión intraventricular.

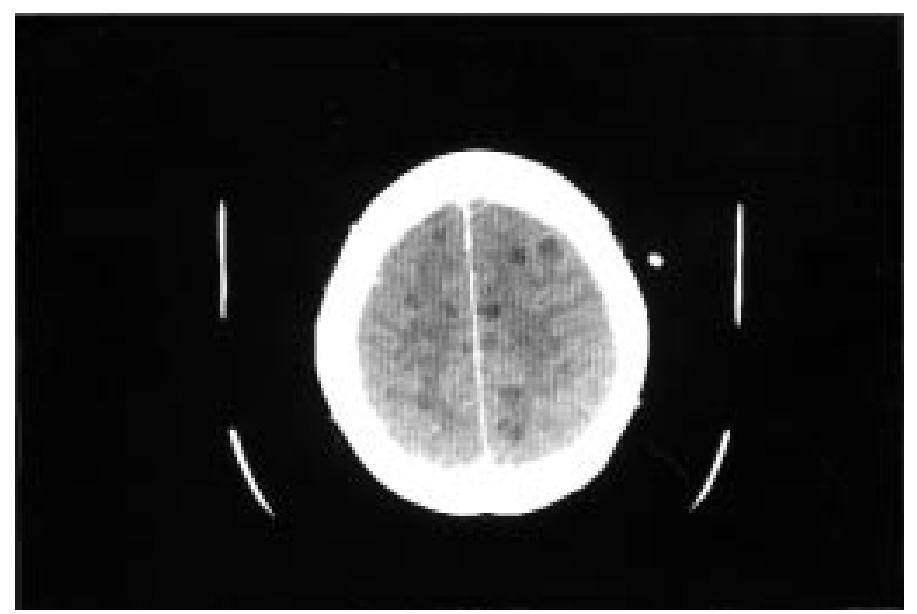

Figura 2. Tomografía computarizada de encéfalo sin contraste. Múltiples lesiones subcorticales hipodensas que corresponden a abscesos de aspergilosis.

aproximadamente 2 meses posterior al trasplante y el diagnóstico fue confirmado con biopsia rinosinusal. Se trató con anfotericina B liposomal, debridamiento rinofaríngeo y esfenoidotomía bilateral con buen resultado clínico.

Cuatro pacientes presentaron MP $(17,4 \%)$, en tres se presentó como síndrome confusional, temblor de extremidades, disartria y dismetría, y en uno se manifestó como un estado epiléptico mioclónico. Uno de los pacientes tenía una hiponatremia de $121 \mathrm{mEq} / \mathrm{L}$ previo al trasplante, los otros tres tenían natremias normales y $\mathrm{C}_{2}$ dentro de rango terapéutico. En todos los casos el diagnóstico fue por los hallazgos en la RM. El tratamiento consistió en la suspensión de ciclosporina, reemplazándola por tacrolimus y micofenolato. Uno de los pacientes requirió tratamiento anticonvulsivante con levetiracetam oral ( $1 \mathrm{~g} \mathrm{c} / 8 \mathrm{~h})$, para el control de las crisis mioclónicas. Uno de los pacientes persistió con disartria leve e inestabilidad de la marcha, el resto se recuperó completamente.

Complicaciones del SNP se observaron en 5 pacientes $(21,7 \%), 1$ presentó una neuralgia secundaria a herpes zoster intercostal, 2 una neuro- 
patía compresiva (uno cubital y otro peronea) y 1 una polineuropatía de fibra fina. Todos los pacientes se recuperaron satisfactoriamente. Un paciente presentó una polineuropatía del paciente crítico, que posteriormente falleció debido a una sobreinfección bacteriana y sepsis secundaria.

En total 5 pacientes con complicaciones neurológicas fallecieron (22\%): 2 debido a una hemorragia encefálica masiva, 2 por aspergilosis encefálica y 1 con polineuropatía del paciente crítico. La mortalidad de los pacientes que no desarrollaron una $\mathrm{CN}$ fue $16 \%$.

La mediana de los días de hospitalización en los pacientes con $\mathrm{CN}$ fue 22 días [15-89], mientras que en los que no desarrollaron una $\mathrm{CN}$ fue 13 días [2-78].

\section{Discusión}

Las CN de los trasplantes se dividen en dos grandes categorías: las comunes a todo tipo de trasplante, principalmente secundarias a tratamiento inmumosupresor y las específicas de cada trasplante, por la patología subyacente o por fenómenos de la técnica del trasplante. Con relación a los THO, las CN específicas incluyen: encefalopatía, enfermedad cerebrovascular, convulsiones, infecciones del SNC, MP y lesión de nervios periféricos ${ }^{10,11}$.

La incidencia de las CN en nuestra serie fue $30,3 \%$ (23 pacientes). Otras series, con un mayor número de pacientes reportan porcentajes que varían entre $24 \%$ y $33 \%$ (12-17). Una serie chilena presenta cifras de $\mathrm{CN}$ de $20 \% 18$.

Se ha descrito que un buen predictor de $\mathrm{CN}$ posterior a un THO es la severidad de la insuficiencia hepática previa a la intervención, señalando que la encefalopatía hepática es el factor más determinante $^{19}$ y que revierte posterior al THO $^{20}$. En nuestra serie observamos que la mayoría de los pacientes que desarrollaron una $\mathrm{CN}$ tenía una insuficiencia hepática moderada 0 avanzada (Child B o C).

Se reportan series en que el uso de ciclosporina se asocia a una $\mathrm{CN}$ en $15 \%$ a $40 \%$ de los pacientes $^{21,22}$. Las más frecuentemente descritas son: convulsiones, compromiso de conciencia y temblor. En nuestro grupo, la neurotoxicidad por ciclosporina correspondió a $30,4 \%$ de las $\mathrm{CN}$.
Existe una diferencia estadísticamente significativa entre las concentraciones plasmáticas de ciclosporina de los pacientes que presentaron esta $\mathrm{CN}$ y la de los que no $(p=0,02)$. Algunos estudios sostienen que la aparición de neurotoxicidad por ciclosporina es más frecuente con niveles plasmáticos elevados, aunque se puede presentar dentro de un amplio rango terapéutico ${ }^{22,23}$. La neurotoxicidad es reversible al retirar o disminuir la dosis del fármaco ${ }^{22,23}$. Observamos una significativa disminución de neurotoxicidad al incorporar $\mathrm{C}_{2}$ para la monitorización de los niveles de ciclosporina, lo que concuenda con la experiencia de otros centros que describen una disminución en la incidencia de rechazo y de toxicidad al utilizar esta técnica de medición ${ }^{24,25}$.

De los pacientes trasplantados, dos $(2,6 \%)$ presentaron un síndrome de hiperperfusión cerebral, manifestada por convulsiones. En series internacionales se describen incidencias de hasta $5 \%{ }^{7,16,26-28}$. Este síndrome consiste en un cuadro reversible de cefalea, alteraciones visuales, somnolencia y convulsiones, asociado a leucoencefalopatía, la que se localiza predominantemente en los lóbulos occipitales en las neuroimágenes ${ }^{29}$. Su patogenia se ha asociado a terapia inmunosupresora con ciclosporina y tacrolimus, encefalopatía hipertensiva y eclampsia ${ }^{29-33}$.

La enfermedad cerebrovacular representa $4 \%$ de las complicaciones en pacientes con $\mathrm{THO}^{26-28,34}$, lo que corresponde a $14 \%$ de las $\mathrm{CN}^{14}$. Los resultados de nuestra serie coinciden con estos datos.

Los 3 pacientes que desarrollaron una hemorragia encefálica estaban siendo tratados con micofenolato y tenían, al momento de la $\mathrm{CN}$, un recuento plaquetario igual o inferior a 40.000/ $\mathrm{mm}^{3}$. Ambos pacientes con hemorragias masivas y que fallecieron estaban recibiendo antiagregantes plaquetarios. No hemos encontrado evidencia de trombocitopenia secundaria al uso de micofenolato. Si bien nuestros pacientes presentaban pruebas de coagulación normal al momento de la $\mathrm{CN}$, se ha reportado que la coagulopatía es la principal causa de hemorragia intracraneana en estos pacientes ${ }^{35}$.

Las infecciones del SNC se presentan habitualmente después del primer mes del THO, siendo el mayor riesgo entre el $1^{\circ}$ y $6^{\circ}$ mes, en este periodo predominan dos tipos de infecciones: virales (citomegalovirus y virus de Epstein Barr) y por organismos oportunistas (Listeria monocytogenes, 
Aspergillus fumigatus y Nocardia asteroides) ${ }^{10,36}$. En una serie intrahospitalaria, la incidencia de infecciones del SNC fue $1 \%{ }^{14}$. Otras series describen hasta $5 \% 15,16,34,37$. En la mayoría de estos casos (73\%) se documenta una infección pulmonar concomitante por el mismo agente patógeno ${ }^{38}$. En nuestra serie se registraron dos casos de aspergilosis encefálica asociado a compromiso pulmonar. Ambos pacientes fallecieron.

Una serie de 50 pacientes fallecidos posterior a THO fueron sometidos a estudio neuropatológico, cinco tenían MP que no fue diagnosticada previamente $^{39}$. No se observó una correlación directa entre cambios bruscos de la concentración de sodio plasmático y la aparición de $\mathrm{MP}^{39}$. Yu et al comunicaron una incidencia de MP de 3,5\%, encontrando en algunos casos una hiponatremia previa al $\mathrm{THO}^{40}$. En nuestra serie tuvimos cuatro pacientes con MP, todos se recuperaron. Esto se puede explicar por el diagnóstico precoz, al cual estábamos sensibilizados, así como a la posibilidad de realizar una RM de encéfalo que confirmaba el diagnóstico. Esto nos llevó a realizar un tratamiento precoz consistente en un manejo prolijo de la natremia y disminución o cambio del inmunosupresor.

Las complicaciones del SNP y su incidencia en pacientes con THO varían ampliamente en la literatura $11,14,21$. De nuestros pacientes, 21,7\% presentó una complicación del SNP. Se reporta que hasta $10 \%$ de los pacientes sometidos a un THO presentan lesiones compresivas de nervios periféricos, el nervio cubital es el más frecuente-

\section{REFERENCIAS}

1. Starzl Te, Marchioro TL, Von Kaula K, Hermann G, BRITAIN R, WADDELL W. Homotransplantation of the liver in humans. Surg Gynecol Obstet 1963; 117: 659-76.

2. StaRzL TE. The failed liver transplant trials. En Starzl T.E. ed.: The puzzle people. Memoirs of a transplant surgeon. Pittsburgh, U.S.: University of Pittsburgh Press, 1992; 96-105.

3. EASL: National Institutes of Health Consensus Development Conference Statement: Liver transplantation-June 20-23, 1983. Hepatology 1984; 4 (1 Suppl): 107S-110S.

4. Bitrán E, Cenitagoya G, Orellana H. Trasplante ortotópico del hígado, un caso de hepatoma. Rev Hosp S Fco de Borja 1969; 4: 96-8. mente comprometido ${ }^{41}$. Dentro de las complicaciones neuromusculares asociadas a THO, también se describe la radiculopatía post-herpes zoster y la tetraplejia secundaria a miopatía 0 neuropatía del paciente crítico ${ }^{43,44}$. Se describe una buena evolución en los sobrevivientes ${ }^{42}$.

Las CN se asocian a una mayor estadía hospitalaria, las series fluctúan entre 12 y 31 días ${ }^{12-14}$, similar a los 22 días de nuestra muestra. Por último, la mortalidad atribuible a una $\mathrm{CN}$ en series internacionales fluctúa entre 16,3\% y 26,1\%12,13,21, similar a $22 \%$ observado en nuestra serie. Si bien algunas series han encontrado una mayor mortalidad en los pacientes que desarrollan una $\mathrm{CN}^{15}$, otras no han encontrado diferencias ${ }^{13,23}$, como ocurre con nuestros pacientes.

\section{ConcLusión}

Las CN son una causa importante de morbimortalidad en paciente con un THO. En nuestra serie encontramos incidencias similares a estudios internacionales. El 30\% desarrolló una $\mathrm{CN}$, de éstos $22 \%$ falleció debido a la CN. La presencia de CN determinó una mayor estadía hospitalaria.

La pesquisa precoz y tratamiento oportuno de algunas $\mathrm{CN}$ puede disminuir la mortalidad como ocurrió en nuestros pacientes con encefalopatía por hiperperfusión, MP y mucormucosis. El uso de $\mathrm{C}_{2}$ para la monitorización de los niveles de ciclosporina puede disminuir la incidencia de neurotoxicidad.

5. Hepr J, Zaror M, Ríos H, Suárez L, Figueroa P, Quiroga M, Rodríguez G. Perspectiva del trasplante hepático en Chile. Rev Méd Chile 1990; 118: 453-8.

6. Martínez J. Implantación con técnica de Piggy-Back y anastomosis cavo-cava lateral en trasplante hepático ortotópico. Experiencia inicial en un Centro Universitario. Rev Chil Cir 2004; 56: 317-31.

7. Strouse T. Neuropsychiatric Outcomes in Liver Transplantation. En: Busuttil R, Klintmalm G, ed. Transplantation of the liver. Philadelphia, Pennsylvania, U.S.: W.B. Saunders Company; 1996; 659-64.

8. Martínez J, Jarufe N, Crovari F, Alvarez S, Arrese M, PÉREZ RM ET AL. Procura de hígado para trasplante hepático ortotópico. Comparación entre dos técnicas. Rev Chil Cir 2005; 57: 26-32.

9. Pugh RN, Murray-Lyon I, Dawson J, Pietroni MC, 
Wiwams R. Transection of the oesophagus for bleeding oesophageal varices. Br J Surg 1974; 60: 646-9.

10. PAтснеш RA. Neurological complications of organ transplantation and immunosuppressive agents. En: Aminoff MJ ed. Neurology and General Medicine. New York. Churchill Livingstone; 2001; 763-75.

11. Piess M, Zivkovic S. Neurologic complications of transplantation. The Neurologist 2002; 8: 107-20.

12. SANer FH, Gu Y, Minouchehr S, ILker K, Fruhauf N, PAUl A ET AL. Neurological complications after cadaveric and living donor liver transplantation. J Neurol 2006; 253: 612-17.

13. Saner FH, Sotiropoulos GC, Gu Y, Paul A, Radtke A, GeNSICKE J ET AL. Severe neurological events following liver transplantation. Arch Med Res 2007; 38: 75-9.

14. Lewis MB, HowdiE PD. Neurologic complications of liver transplantation in adults. Neurology 2003; 61: 1174-8.

15. Pujol A, Graus F, Rimola A, Beltrán J, GarcíaValdecasas JC, Navasa M et al. Predictive factors of inhospital CNS complications following liver transplantation. Neurology 1994; 44: 1226-30.

16. Adams DH, Ponsford S, Gunson B, Boon A, Honigberger L, Wiшams ET AL. Neurological complications following liver transplantation. Lancet 1987; 1: 949-51.

17. Guarino M, Stracciari A, Pazzagla P, Sterzi R, Santim I, Donato F, D'AIESSANDRO R. Neurological complications of liver transplantation. J Neurol 1996; 243: 137-42.

18. Hepp J, Ríos H, Sú́rez L, Zaror M, Quiroga M, RodríGuez G ET AL. Trasplante hepático en adultos: casuística de Clínica Alemana de Santiago. Rev Méd Chile 2002; 130: 779-86.

19. Dhar R, Young GB, Marotta P. Perioperative neurological complications after liver transplantation are best predicted by pre-transplant hepatic encephalopathy. Neurocrit Care 2008; 8: 253-8.

20. Mattarozzi K, Stracciari A, Vignatem L, D’Aiessandro R, Moreu M, Guarino M. Minimal hepatic encephalopathy longitudinal effects of liver transplantation. Arch Neurol 2004; 61: 242-7.

21. Kim BS, Lee SG, Hwang S, Park KM, Kim KH, Ahn CS et AL. Neurologic complications in adult living donor liver transplant recipients. Clin Transplant 2007; 21: 544-7.

22. Gijtenbeek JM, Van Den Bent MJ, Vecht CJ. Cyclosporine neurotoxicity: a review. J Neurol 1999; 246: 339-46.

23. WiJdicKs EF, WiesNeR RH, KROM RA. Neurotoxicity in liver transplant recipients with cyclosporine immunosuppression. Neurology 1995; 45: 1962-4.

24. LEVY GA. Neoral C2 in liver transplant recipients. Transplant Proc 2001; 33: 3089-91.

25. Santoyo J, Suárez MA, Pérez-Daga Ja, Fernández JL, RodRIGo J, JiMÉNEZ M. Efficacy of C2 monitoring of cyclosporine neoral in adult liver transplantation: a comparative study. Transplant Proc 2001; 33: 3096-7.

26. Vogt DP, Lederman RJ, Carey WD, Broughan TA. Neurologic complications of liver transplantation. Transplantation 1988; 45: 1057-61.
27. Moreno E, Gómez SR, González I, Loinaz C, García I, Pérez A ET AL. Neurologic complications in liver transplantation. Acta Neurol Scand 1993; 87: 25-31.

28. Stein DP, Lederman RJ, Vogt DP, Carey WD, Broughan TA. Neurological complications following liver transplantation. Ann Neurol 1992; 31: 644-9.

29. Sandoval P, Retamal E. Encefalopatías por hiperperfusión encefálica. Cuadernos de Neurología 2006; 5-26.

30. Nakamura M, Fuchinoue S, Sato S, Hoshino T, Samada T, SAGESHIMA J ET AL. Clinical and radiological features of two cases of tacrolimus-related posterior leukoencephalopathy in living related liver transplantation. Transplant Proc 1998; 30: 1477-8.

31. Hinchey J, Chaves C, Appignani B, Breen J, Pao L, Wang A ET AL. A reversible posterior leukoencephalopathy syndrome. N Engl J Med 1996; 334: 949-50.

32. Fruhauf NR, Koeppen S, Saner FH, Egelhof T, Stravrou G, NADALIN S ET AL. Late onset of tacrolimus-related posterior leukoencephalopathy after living donor liver transplantation. Liver Transpl 2003; 9: 983-5.

33. Lavigne CM, Shrier DA, KetKar M, Powers JM. Tacrolimus leukoencephalopathy: A neuropathologic confirmation. Neurology 2004; 63: 1132-3.

34. Menegaux F, Keefre EB, Andrews BT, Egawa H, Monge $\mathrm{H}$, ConCEPCION W ET aL. Neurological complications of liver transplantation in adult versus pediatric patients. Transplantation 1994; 58: 447-50.

35. Estol CJ, Pessin MS, Martínez AJ. Cerebrovascular complications after orthotopic liver transplantation: a clinicopathologic study. Neurology 1991; 41: 815-9.

36. ConTi DJ, RuBin RH. Infection of the central nervous system in organ transplant recipients. Neurol Clin 1988; 6: 241-60.

37. FerReiro JA, Robert MA, Townsend J, VinTeR HV. Neuropathologic findings after liver transplantation. Acta Neuropathol 1992; 84: 1-14.

38. EMmanouildes C, Holtc, Winston D. Infections after liver transplantation. En: Busuttil R, Klintmalm G, ed. Transplantation of the liver. Philadelphia, Pennsylvania, U.S.: W.B. Saunders Company; 1996; 633-47.

39. Boon AP, Carey MP, Adams DH, Buckels J, MCMaster P. Central pontine myelinolisis in liver transplantation. J Clin Pathol 1991; 44: 909-14.

40. Yu J, Zheng S, Lang T, Shen Y, Wang W, Ke Q. Possible causes of central pontine myelinolysis after liver transplantation. World J Gastroenterol 2004; 10: 2540-3.

41. Campelone JV, Lacomis D, Giulani MJ, Kramer DJ. Mononeuropathies associated with liver transplantation. Muscle Nerve 1998; 21: 896-901.

42. Wijdicks EF, Ltchy WJ, Wiesner RH, KROM RA. Neuromuscular complications associated with liver transplantation. Muscle Nerve 1996; 19: 696-700.

43. Latronico N, Peli E, Botteri M. Critical illness myopathy and neuropathy. Curr Opin Crit Care 2005; 11: 126-32. 